Hydroécol. Appl. (1994) Tome 6 Vol 1-2, pp. 293-314

\title{
Influence de la vidange d'une retenue sur certaines caractéristiques physiologiques chez divers Téléostéens
}

\author{
Impact of draining of a reservoir on certain physiological \\ characteristics of some Teleosts
}

\section{F. Bau, J.P. Parent et F. Vellas}

Laboratoire d'Hydrobiologie (CNRS, URA 695), Université Paul Sabatier, 118, route de Narbonne, F-31062 Toulouse Cedex, France.

Résumé. - Lors de la vidange de la retenue de Pareloup, on pouvait supposer que la restriction de l'espace vital entrainerait des modifications de la biologie et de la physiologie des poissons. Dans le but d'obtenir des données sur l'état "sanitaire" des populations pisciaires, l'évolution de caractéristiques morphophysiologiques (facteur de condition, rapports gonado- et hépatosomatiques) et de quelques paramètres sanguins (hématocrite, glycémie, protéinémie, lipémie et insulinémie) a été suivie. Les campagnes de pêches se sont déroulées d'avril à juillet 1992 et lors de la vidange progressive de la retenue, d'avril à juin 1993. Les prises de sang par ponction intracardiaque ont été effectuées dès la capture d'espèces carnivores (brochet, sandre, perche) et omnivores (brème, chevesne). De cette étude, il ressort que :

- les poissons, et en particulier le sandre, présentent une forte valeur de la glycémie consécutive au stress occasionné par la pêche aux filets,

- le comportement alimentaire des animaux au moment de la vidange est différent selon l'espèce considérée,

- mais la variabilité du volume de la retenue ne semble pas avoir d'incidence pathologique majeure sur le métabolisme des poissons.

Mots-clés. - Téléostéens, retenue, vidange, écophysiologie.

Abstract. - At the time of draining of Pareloup reservoir, it was assumed that restriction of living space would lead to biological and physiological changes in fish. In order to obtain data on the state of health of fish populations, changes in physiological characteristics (condition factor, gonado- and hepatosomatic index) and in some blood parameters (hematocrit, glucose, proteins, lipids and insulin) were monitored. Field experiments were conducted from April to July 1992, and during the gradual draining 
of the reservoir from April to June 1993. Blood samples were taken by intracardiac puncture immediately following netting of carnivorous (pike, pikeperch, perch) and omnivorous (bream, chub) species. The study indicated that:

- fish, in particular pikeperch, show high glucose levels following the stress of being netted,

- feeding behavior at the time of the draining differs according to species,

- however, the varying volume of water in the reservoir does not appear to have a major pathological impact on fish metabolism.

Key-words. - Teleosts, reservoir, draining, ecophysiology.

\section{INTRODUCTION}

La vidange de la retenue de Pareloup (Massif Central, France), trente ans après la mise en eau, a permis de réaliser une étude sur l'incidence de la diminution du volume du lac sur la physiologie des poissons. Depuis une dizaine d'années, le laboratoire a consacré une part importante de ses travaux à l'écophysiologie de la perche et du gardon vivant dans cette retenue mésotrophe. Aussi, est-il apparu intéressant de suivre, chez divers Téléostéens carnivores et omnivores, les variations de certaines caractéristiques morphophysiologiques et de paramètres sanguins au cours du printemps 1992 et de les comparer à celles observées au printemps 1993, lors de la vidange progressive du bassin.

\section{MATÉRIEL ET MÉTHODES}

Les animaux sont capturés, chaque mois, à l'aube et au crépuscule, à l'aide de filets de maille $70 \mathrm{~mm}$, posés durant 2 heures; ces filets permettent une capture sélective d'individus de grande taille. Lors de la vidange (14-
18 juin 1993), les poissons sont directement récupérés à la pêcherie en aval du barrage. La ponction intracardiaque est aussitôt effectuée, l'animal étant maintenu en immersion. Le sang est recueilli sur héparine. Après l'évaluation de l'hématocrite, le sang est centrifugé. Le plasma est ensuite rapidement congelé dans l'azote liquide et conservé à $-80^{\circ} \mathrm{C}$. Les poissons sont ensuite sacrifiés puis mesurés (poids, longueur standard) pour déterminer le facteur de condition, les gonades et le foie étant également prélevés et pesés pour établir respectivement les rapports gonado- et hépatosomatiques. Au laboratoire, le glucose, le cholestérol et les triglycérides plasmatiques sont déterminés de façon enzymatique (test bioMérieux), les protéines totales étant dosées par une méthode colorimétrique selon une réaction du type Biuret (kit bioMérieux). Le taux d'insuline du plasma est évalué par un dosage radioimmunologique (SB-INSI-5, Cis bio International), utilisant la méthode des doubles anticorps de Morgan et Lazarow (1963).

L'analyse statistique des résultats est effectuée par le t-test de Student (Statwork, Macintosh). 


\section{RÉSULTATS}

Lors de la première période, d'avril 1992 à juillet 1992, le volume de la retenue de Pareloup était de l'ordre de 100 à 150 millions de $\mathrm{m}^{3}$ d'eau. $\mathrm{A}$ partir du mois de décembre, la vidange du lac a été progressivement entreprise, amenant à 20 millions de $\mathrm{m}^{3}$ le volume de la retenue en avril 1993, puis à 2 millions de $\mathrm{m}^{3}$ début juin et enfin à 0,3 millions de $\mathrm{m}^{3}$ juste avant la vidange (tableau I).

Les captures des poissons sont rapportées dans le tableau II. Un problème d'échantillonnage s'est posé lors de ces travaux: certaines espèces n'ont pu être pêchées de façon régulière. Aussi, chez les omnivores, seuls les résultats concernant la brème et le chevesne ont-ils été donnés.

\section{- Caractéristiques morphophysio- logiques}

Rapport gonadosomatique (tableau III) (RGS = poids des gonades $(\mathrm{g})$ * $100 /$ (poids total - poids des gonades) (g)).

En 1992, la brème et le chevesne ont frayé en juin et les Percidés et le brochet en mai. En 1993, les périodes de fraie sont identiques à celles observées en 1992 chez la brème et les Percidés, alors que l'émission des produits génitaux est plus tardive chez le brochet et difficile à déterminer chez le chevesne.

Facteur de condition (fig. 1)

$(F C=$ poids total $(g) * 100 /$ (longueur standard $\left.)^{3}(\mathrm{~cm})\right)$.

Tableau I. - Données physicochimiques relevées au cours de deux périodes dans la retenue de Pareloup.

Table I. - Physicochemical data obtained during two periods in Pareloup reservoir.

\begin{tabular}{|c|c|c|c|c|c|c|c|c|}
\hline & $\begin{array}{l}\text { Avril } 1992 \\
\text { (7/04/1992) }\end{array}$ & $\begin{array}{c}\text { Mai 1992 } \\
(4 / 05 / 1992)\end{array}$ & $\begin{array}{c}\text { Juin } 1992 \\
(1 / 06 / 1992)\end{array}$ & $\begin{array}{l}\text { Juillet } 1992 \\
(2 / 07 / 1992)\end{array}$ & $\begin{array}{c}\text { Avril } 1993 \\
(13 / 04 / 1993)\end{array}$ & $\begin{array}{c}\text { Mai 1993 } \\
(5 / 05 / 1993)\end{array}$ & $\begin{array}{c}\text { Juin } 1993 \\
(8 / 06 / 1993)\end{array}$ & $\begin{array}{c}\text { Vidange } \\
(14 / 06 / 1993)\end{array}$ \\
\hline $\begin{array}{l}\text { Volume de la } \\
\text { retenue }\left(\mathrm{m}^{3}\right)\end{array}$ & $100-150.10^{6}$ & $100-150.10^{6}$ & $100-150.10^{6}$ & $100-150.10^{6}$ & $20.10^{6}$ & $20.10^{6}$ & $2.10^{6}$ & $0,3.10^{6}$ \\
\hline \multicolumn{9}{|l|}{ Température $\left({ }^{\circ} \mathrm{C}\right)$} \\
\hline à: $-0 \mathrm{~m}$ & 10 & 14 & 17 & 18 & 7 & 11,5 & 14,5 & 15 \\
\hline$:-10 \mathrm{~m}$ & 7 & 11 & 16 & 17 & 6,5 & 7,5 & 11 & 12 \\
\hline $\begin{array}{l}\text { Oxygène dissous } \\
\text { (mg/l) }\end{array}$ & $8-11,5$ & $9,5-11$ & $9-11$ & $8,5-9,5$ & $10-14$ & $10-11$ & $10-11$ & $8,5-10,9$ \\
\hline $\begin{array}{l}\text { Alcalinité } \\
\text { (mg CaCo }\end{array}$ & 35 & 34 & 27 & 22 & 23 & 21 & 33 & - \\
\hline $\begin{array}{l}\text { Phosphore total } \\
(\mu \mathrm{g} / 1)\end{array}$ & 12,5 & 15,5 & 13,5 & 11 & 32 & 29 & 48 & - \\
\hline Nitrates (mg N/l) & 1,62 & 1,65 & 1,66 & 1,60 & 1,60 & 1,69 & 1,47 & - \\
\hline $\begin{array}{l}\text { Chlorophylle a } \\
(\mu \mathrm{g} / \mathrm{l})\end{array}$ & 8,7 & 7,7 & 1,2 & 3,3 & 8,7 & 9,4 & 7,7 & - \\
\hline
\end{tabular}


Tableau II. - Nombre de poissons retenus lors des campagnes de pêches.

Table Il. - Number of netted fish required for sampling.

\begin{tabular}{|l|c|c|c|c|}
\cline { 2 - 5 } \multicolumn{1}{c|}{} & Avr-92 & Mai & Juin & Juillet \\
\hline Brochet & 13 & 3 & 4 & 3 \\
\hline Sandre & 4 & 4 & 6 & 6 \\
\hline Perche & 3 & - & 10 & 3 \\
\hline
\end{tabular}

\begin{tabular}{|c|c|c|c|}
\hline Avr-93 & Mai & Juin & Vidange \\
\hline 9 & 12 & 7 & 7 \\
\hline 7 & 6 & 3 & 7 \\
\hline 6 & 9 & 8 & 7 \\
\hline
\end{tabular}

\begin{tabular}{|l|c|c|c|c|}
\hline Brème & 8 & 7 & 7 & 8 \\
\hline Chevesne & 3 & 3 & 3 & 3 \\
\hline Tanche & - & - & 4 & 1 \\
\hline Carpe & - & 2 & - & 4 \\
\hline Rotengle & - & - & 2 & - \\
\hline Vandoise & - & - & - & - \\
\hline Gardon & 8 & - & - & - \\
\hline
\end{tabular}

\begin{tabular}{|r|r|r|r|}
\hline 10 & 10 & 11 & 7 \\
\hline 6 & 3 & 3 & 3 \\
\hline 2 & 1 & 5 & 7 \\
\hline- & - & 2 & 2 \\
\hline- & 9 & 3 & - \\
\hline 2 & - & 1 & - \\
\hline- & - & - & - \\
\hline
\end{tabular}

Le facteur de condition est compris entre $0,75-1,0 \%$ chez le brochet, 1,0 $1,35 \%$ chez le sandre, $1,5-2,25 \%$ chez la perche, $1,75-2,0 \%$ chez la brème et $2,0-3,0 \%$ chez le chevesne. Au sein d'une même espèce, ce rapport reste relativement stable au cours de l'étude. Cependant, en avril 1993, les carnassiers et la brème ont des valeurs supérieures à celles trouvées à la même époque en 1992, et en juin 1993, seule une baisse de ce paramètre est notée chez la brème au moment de la vidange.

\section{Rapport hépatosomatique (fig. 2)}

$(\mathrm{RHS}=$ poids du foie $(\mathrm{g}) * 100 /$ (poids total - poids des gonades) (g)).

Une baisse du rapport hépatosomatique est constatée chez le sandre d'avril à juillet 1992 et d'avril à juin 1993, la valeur n'augmentant qu'au moment de la vidange, alors que chez le brochet et la perche, ce paramètre présente une forte variabilité $(0,98$ $2,37 \%$ ) lors des deux périodes. Toutefois, chez ces deux espèces, une baisse est observée en mai. Chez les

Fig. 1. - Evolution du facteur de condition (FC) au cours de deux périodes.

Les valeurs présentées sont des moyennes \pm écart-types; (a), (b), (c)... les lettres différentes indiquent, pour une même espèce, des valeurs significativement différentes $(p \leq 0,05)$ entre les mois d'une même période et pour chacun des mois, $\left(^{*}\right)$ indique une différence significative $(p \leq$ 0,05 ) entre les deux périodes.

Fig. 1. - Changes in the condition factor (CF) during two periods.

Values given are means $\pm S D$; (a), (b), (c)... dissimilar superscripts indicate, within a species, significant differences $(p \leq 0,05)$ between months of a same period and for each month, $\left(^{*}\right)$ denotes a significant difference $(p \leq 0,05)$ between the two periods. 

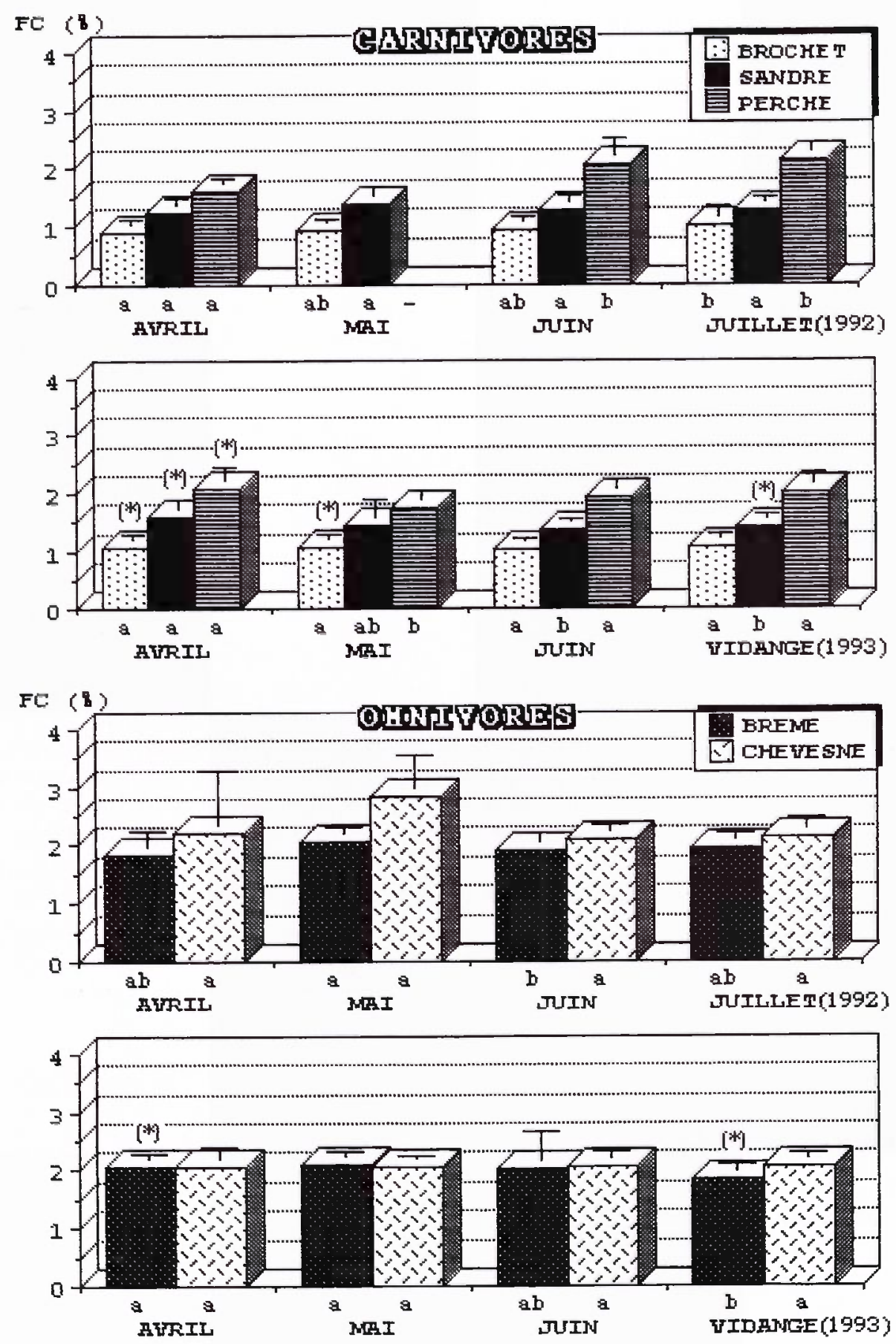
Tableau III. - Valeurs du rapport gonadosomatique (\%) au cours de deux périodes. Les valeurs présentées sont des moyennes \pm écart-types.

Table III. - Gonado-somatic index values (\%) during two periods. Values given are means \pm standard deviation.

\begin{tabular}{|c|lc|c|c|c|}
\cline { 3 - 6 } \multicolumn{2}{c|}{} & Avr-92 & Mai & Juin & Juillet \\
\hline \multirow{3}{*}{ Brochet } & Mâles & $0,9 \pm 0,3$ & 1,63 & $0,7 \pm 0,2$ & 0,59 \\
\cline { 2 - 6 } & Femelles & $9,2 \pm 9,1$ & $0,9 \pm 0,2$ & $0,8 \pm 0,1$ & $0,6 \pm 0,5$ \\
\hline
\end{tabular}

\begin{tabular}{|c|c|c|c|}
\hline Avr-93 & Mai & Juin & Vidange \\
\hline $3,1 \pm 0,7$ & $3,5 \pm 0,7$ & $0,8 \pm 1,2$ & $0,8 \pm 0,7$ \\
\hline $27,1 \pm 4,0$ & $22,4 \pm 5,6$ & - & $6,4 \pm 6,0$ \\
\hline
\end{tabular}

\begin{tabular}{|c|l|r|c|c|c|}
\hline \multirow{3}{*}{ Sandre } & Māles & $0,9 \pm 0,3$ & - & $1,0 \pm 0,4$ & $1,1 \pm 1,4$ \\
\cline { 2 - 6 } & Femelles & $13,5 \pm 3,5$ & $4,9 \pm 4,8$ & - & $2,8 \pm 1,5$ \\
\hline
\end{tabular}

\begin{tabular}{|r|c|c|c|}
\hline $1,1 \pm 0,7$ & 0,89 & $0,6 \pm 0,7$ & $0,5 \pm 0,1$ \\
\hline $15,8 \pm 4,4$ & $3,2 \pm 4,2$ & 1,96 & $2,8 \pm 0,8$ \\
\hline
\end{tabular}

\begin{tabular}{|c|l|c|c|c|c|}
\hline \multirow{2}{*}{ Perche } & Mâles & - & - & 0,86 & 0,13 \\
\cline { 2 - 6 } & Femelles & $29,7 \pm 6,0$ & - & $1,0 \pm 0,1$ & $0,8 \pm 0,1$ \\
\hline
\end{tabular}

\begin{tabular}{|c|c|c|c|}
\hline $3,7 \pm 0,9$ & 2,2 & 0,88 & - \\
\hline $22,4 \pm 18,4$ & $4,8 \pm 1,1$ & $1,6 \pm 0,5$ & $1,5 \pm 0,2$ \\
\hline
\end{tabular}

\begin{tabular}{|l|l|r|r|r|r|}
\hline \multirow{3}{*}{ Brème } & Mâles & $1,7 \pm 0,2$ & $3,2 \pm 0,4$ & $2,0 \pm 0,2$ & $1,0 \pm 0,3$ \\
\cline { 2 - 6 } & Femelles & $2,5 \pm 0,5$ & $13,7 \pm 3,4$ & $9,5 \pm 0,7$ & $4,4 \pm 1,3$ \\
\hline
\end{tabular}

\begin{tabular}{|r|r|r|r|}
\hline $2,8 \pm 0,6$ & $3,5 \pm 0,3$ & $2,4 \pm 0,5$ & $2,0 \pm 0,5$ \\
\hline $14,6 \pm 1,5$ & $15,3 \pm 2,9$ & $9,1 \pm 4,2$ & $8,0 \pm 7,2$ \\
\hline
\end{tabular}

\begin{tabular}{|c|l|c|c|c|c|}
\hline \multirow{2}{*}{ Chevesne } & Mâles & $2,3 \pm 0,3$ & 2,56 & 8,22 & - \\
\cline { 2 - 6 } & Femelles & 19,22 & $15,5 \pm 6,5$ & $7,5 \pm 0,6$ & $7,0 \pm 0,8$ \\
\hline
\end{tabular}

\begin{tabular}{|c|c|c|c|}
\hline 4,67 & 5,44 & 5,66 & - \\
\hline $7,1 \pm 1,8$ & $6,1 \pm 0,9$ & $6,0 \pm 2,0$ & $12,5 \pm 1,3$ \\
\hline
\end{tabular}

omnivores, l'évolution est différente selon l'année considérée: en 1992, les valeurs du rapport hépatosomatique sont nettement élevées en mai, tandis qu'en mai 1993, sont observées les valeurs les plus faibles.

\section{- Paramètres sanguins}

\section{Hématocrite (fig. 3)}

Chez les Percidés, il n'y a pas de variations notables de l'hématocrite au cours des deux périodes étudiées. Chez le brochet, aucune modification de ce paramètre n'est relevée en 1992, alors qu'il est élevé en avril/mai 1993. Chez les omnivores, l'hématocrite est constant et également comparable entre les deux années; chez le chevesne, cependant, la valeur la plus faible est observée en juin 1993 et la plus forte au moment de la vidange.

\section{Glycémie (fig. 4)}

Le sandre montre de très fortes valeurs de la glycémie au cours des deux années $(2,95-6,23 \mathrm{~g} / \mathrm{l})$, les te-

Fig. 2. - Evolution du rapport hépatosomatique (RHS) au cours de deux périodes. (Annotations, voir Fig. 1).

Fig. 2. - Changes in the hepatosomatic index (HSI) during two periods. (For details, see also Fig. 1). 
Influence de la vidange de Pareloup sur la physiologie de divers Téléostéens 299
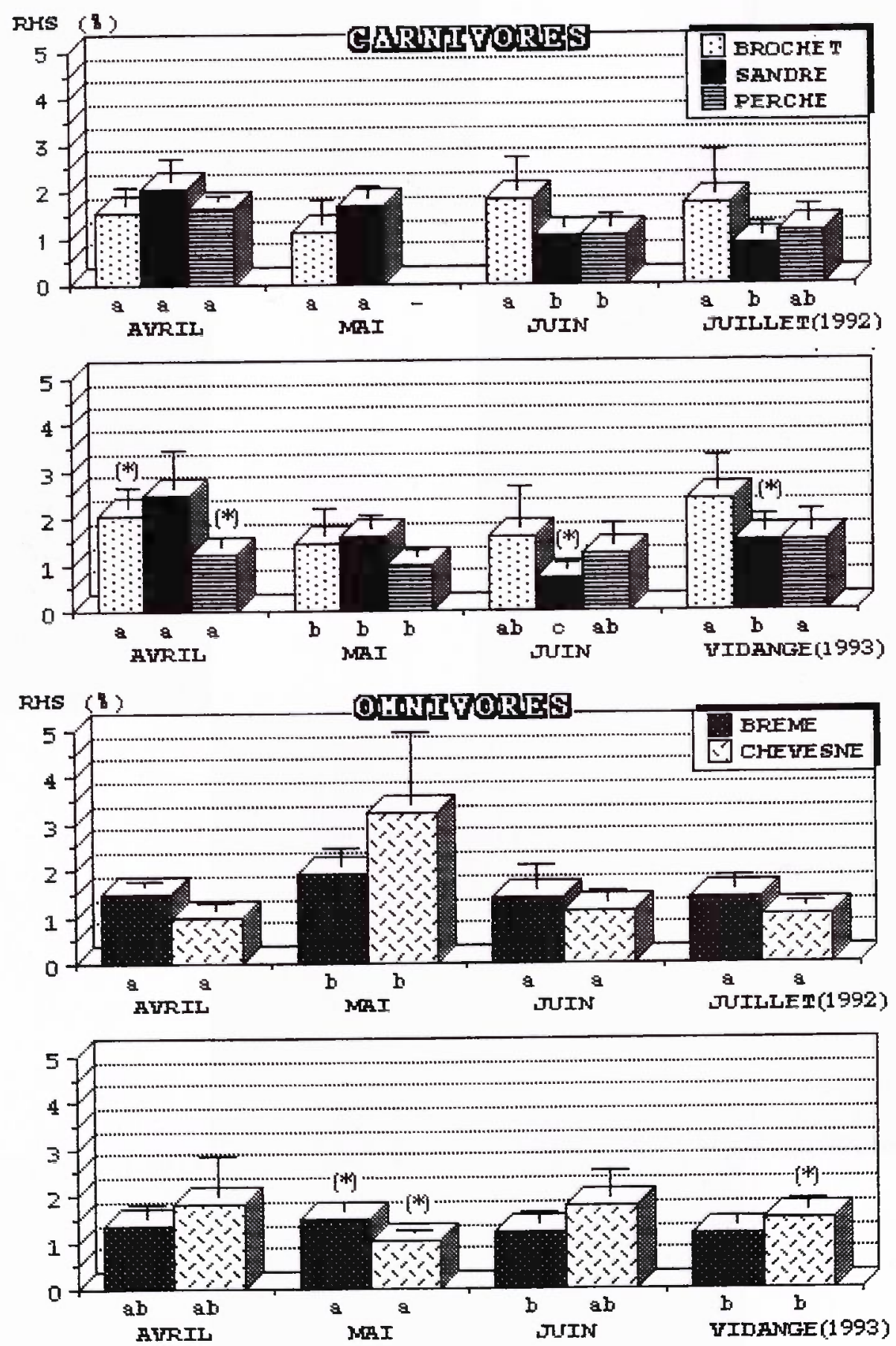

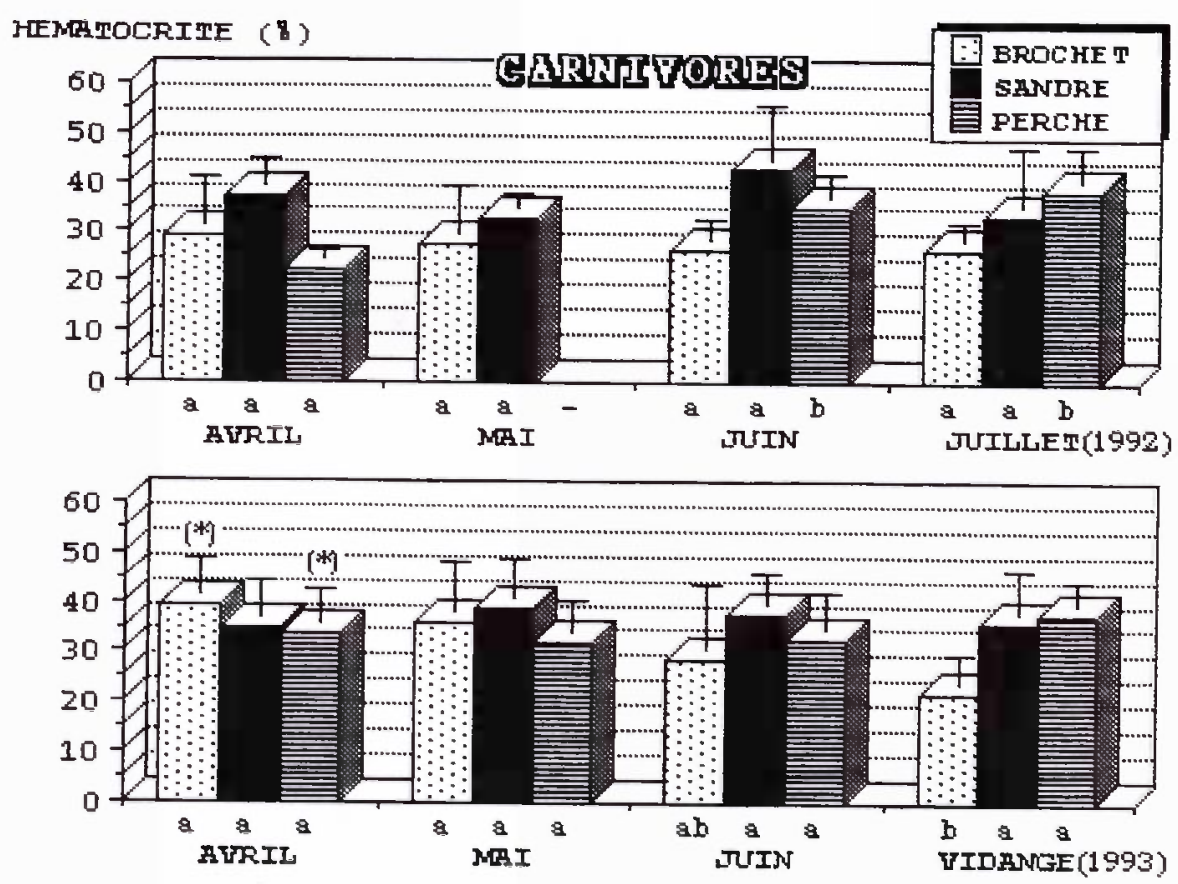

HEMRTOCRITE (B)
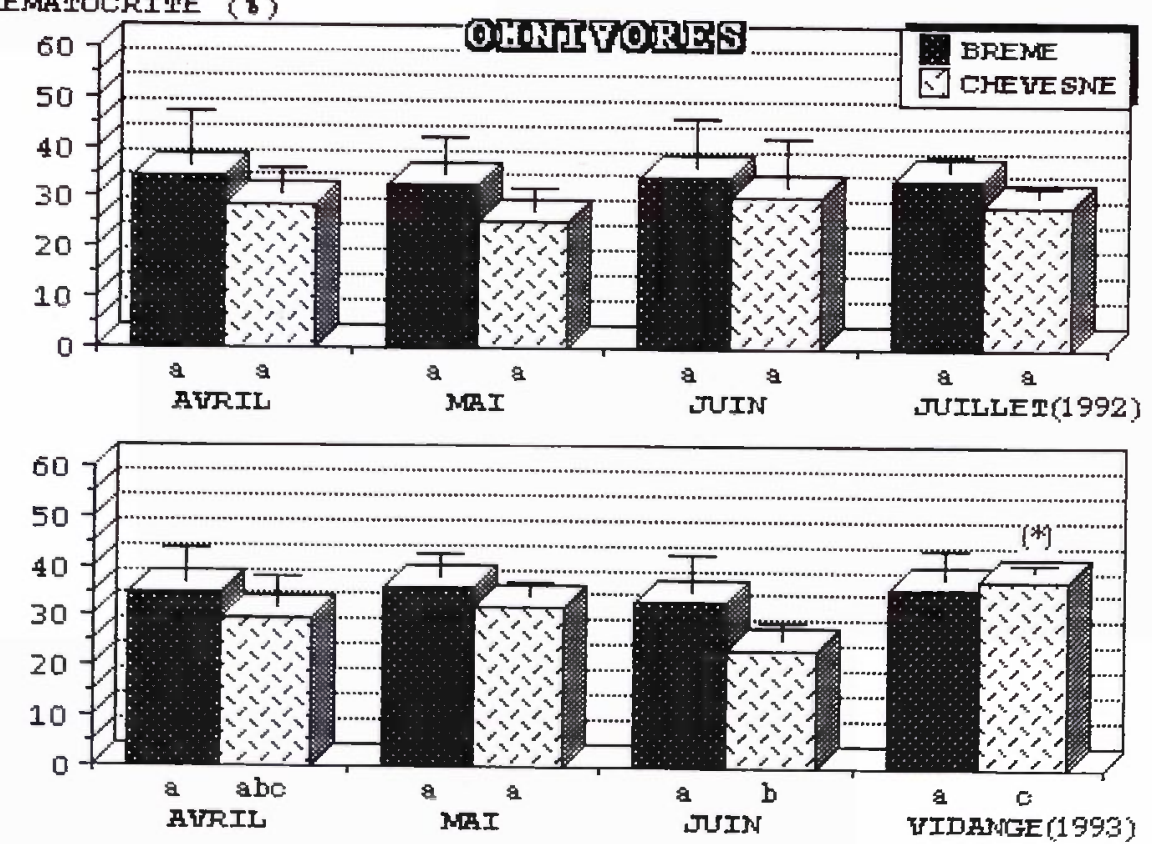

Fig. 3. - Evolution de l'hématocrite au cours de deux périodes. (Annotations, voir Fig. 1).

Fig. 3. - Changes in haematocrit values during two periods. (For details, see also Fig. i). 

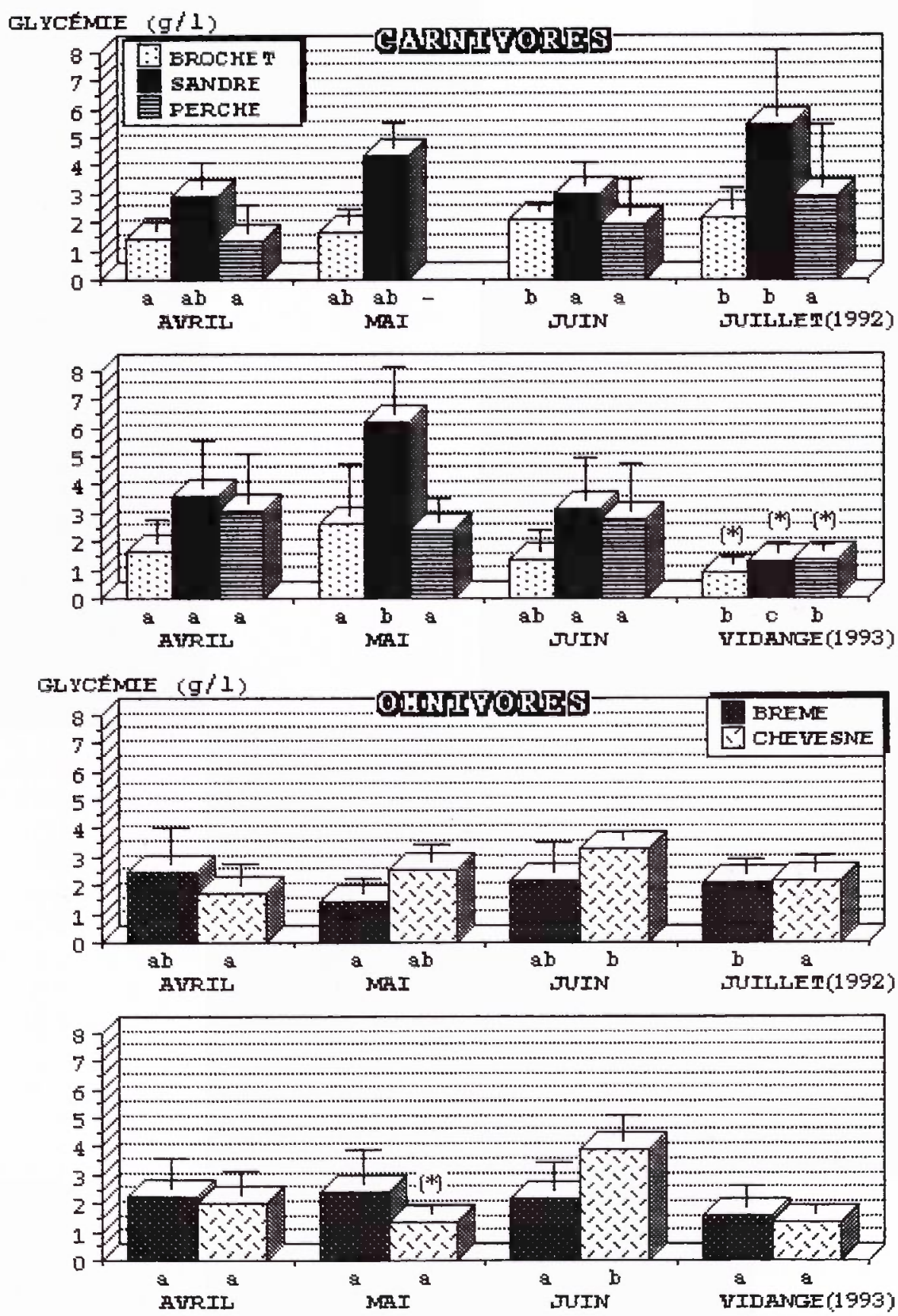

Fig. 4. - Evolution de la glycémie au cours de deux périodes. (Annotations, voir Fig. 1).

Fig. 4. - Changes in plasma glucose levels during two periods. (For details, see also Fig. 1). 
neurs les plus basses étant relevées au moment de la vidange (1,29 \pm $0,37 \mathrm{~g} / \mathrm{l})$. Chez la perche et le brochet, il n'y a pas de variations de ce paramètre physiologiquement significatives en 1992 et l'on retrouve également des teneurs en glucose plasmatique faibles au moment de la vidange. Aucune variation importante de la glycémie n'est observée chez la brème lors des deux périodes d'étude, et les seuls changements notables présentés par le chevesne correspondent à la hausse de ce facteur enregistrée au mois de juin quelle que soit l'année.

\section{Protéinémie (fig. 5)}

Chez le brochet, la protéinémie n'est pas significativement modifiée au cours des deux années. Chez les Percidés et les omnivores, excepté la baisse observée en avril 1992, les valeurs de ce paramètre ne présentent également pas de variations sensibles.

\section{Cholestérolémie (fig. 6)}

Chez le brochet, aucune modification de la cholestérolémie n'apparaît nettement. II en est de même chez les omnivores où les variations de ce paramètre sont peu significatives, excepté la valeur élevée obtenue en avril 1993 chez le chevesne. Chez le sandre, seule une augmentation de la cholestérolémie est notée au moment de la vidange, et chez la perche, la teneur la plus faible en cholestérol plasmatique est enregistrée en avril 1992.

\section{Triglycéridémie (fig. 7)}

Le brochet montre des taux de triglycérides plasmatiques importants en juin quelle que soit l'année considérée. La triglycéridémie évolue, chez la perche, de la même manière que la cholestérolémie, et chez le sandre, les valeurs ne sont pas physiologiquement différentes entre l'année précédant la vidange et le moment même de la vidange, contrairement à ce que l'on observe chez les omnivores. Les teneurs en triglycérides du plasma diminuent en avril 1993 chez la brème et en mai/juin 1993 chez le chevesne, alors que cette dernière espèce présente en mai 1992 les teneurs les plus élevées.

\section{Insulinémie (fig. 8)}

En 1992, le brochet et le sandre ne présentent pas de modifications significatives de l'insulinémie, tandis que chez la perche et les omnivores, les valeurs les plus fortes sont observées en avril. En 1993, les teneurs en insuline plasmatique sont les plus faibles en juin chez toutes les espèces, excepté chez la brème. Les plus fortes valeurs sont enregistrées au moment de la vidange chez l'ensemble des carnassiers, alors que cette hausse ne se retouve pas chez les omnivores.

\section{DISCUSSION}

La vidange de la retenue de Pareloup a été entreprise dès le mois de décembre 1992, le volume du bassin at- 

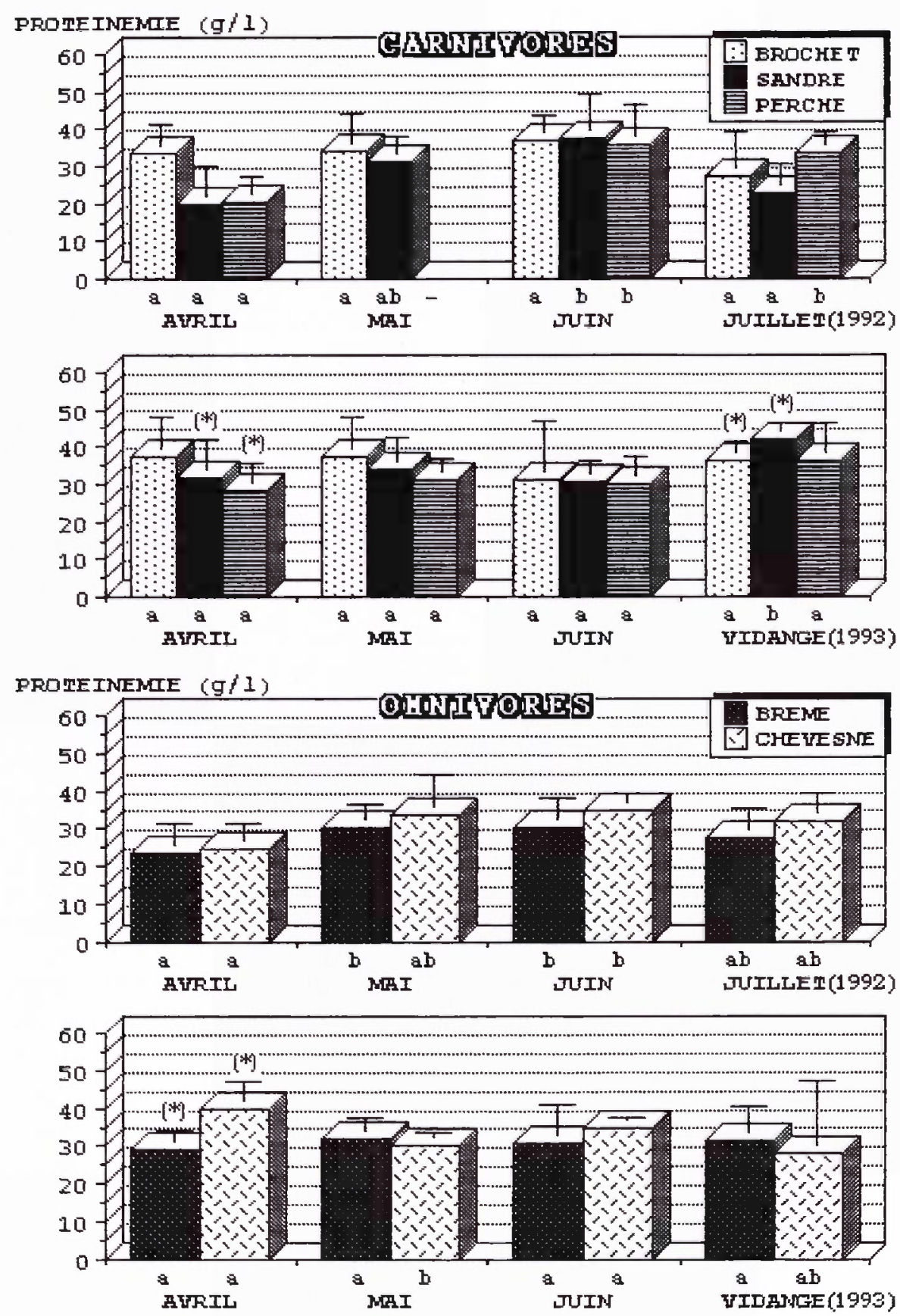

Fig. 5. - Evolution de la protéinémie au cours de deux périodes. (Annotations, voir Fig. 1).

Fig. 5. - Changes in plasma protein levels during two periods. (For details, see also Fig. 1). 

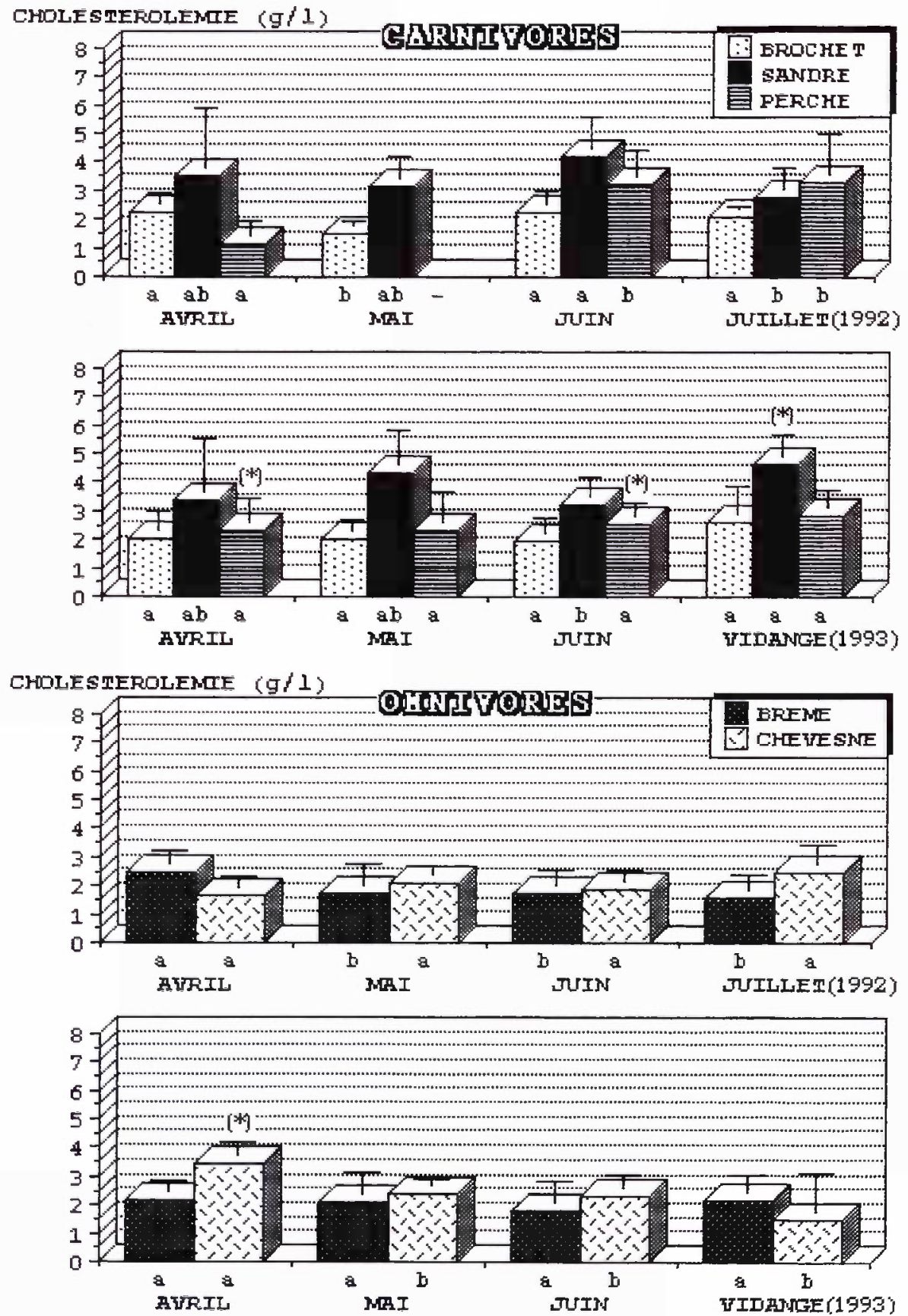

Fig. 6. - Evolution de la cholestérolémie au cours de deux périodes. (Annotations, voir Fig. 1). Fig. 6. - Changes in plasma cholesterol levels during two periods. (For details, see also Fig. 1). 

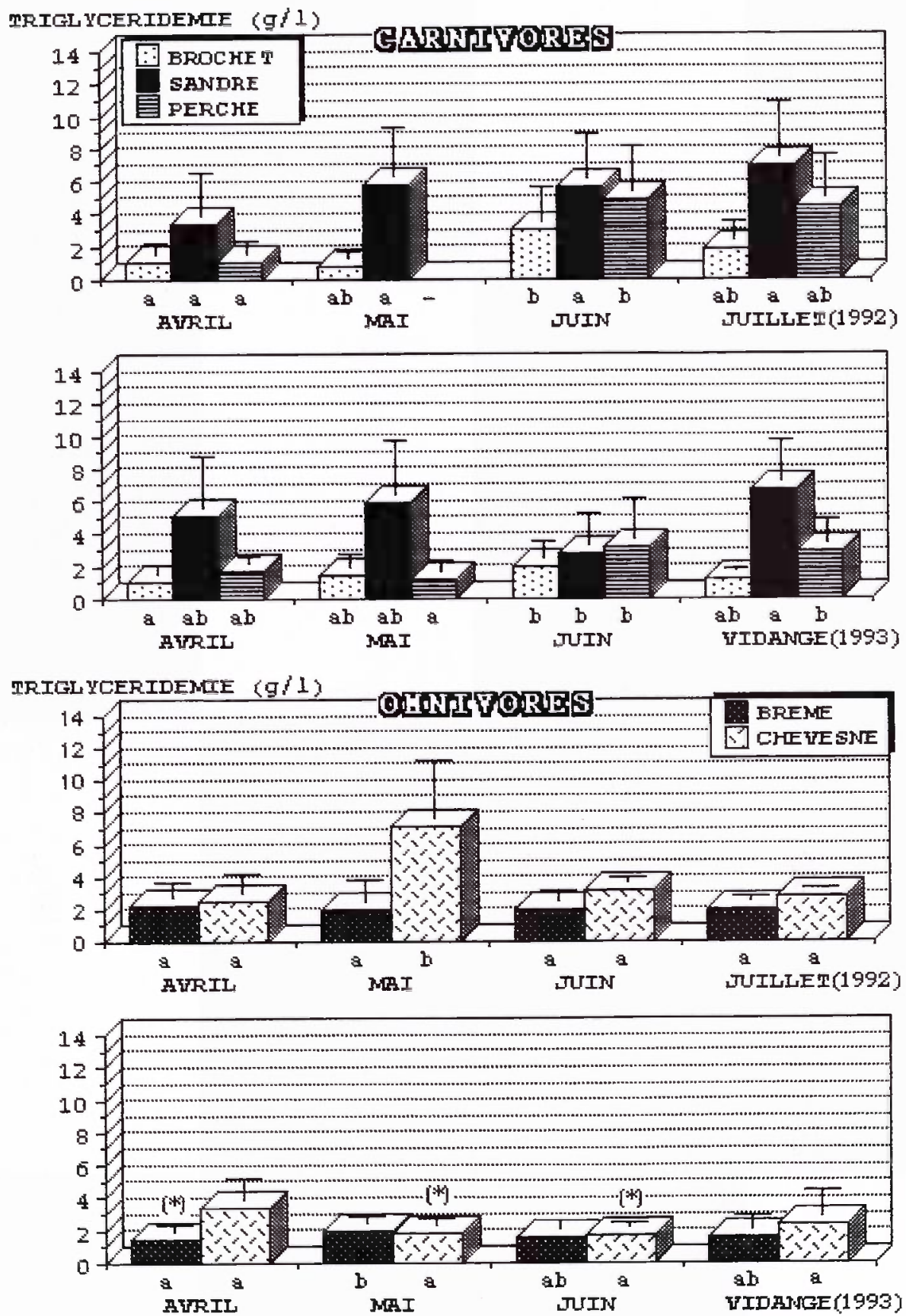

Fig. 7. - Evolution de la triglycéridémie au cours de deux périodes. (Annotations, voir Fig. 1). Fig. 7. - Changes in plasma triglyceride levels during two periods. (For details, see also Fig. 1). 

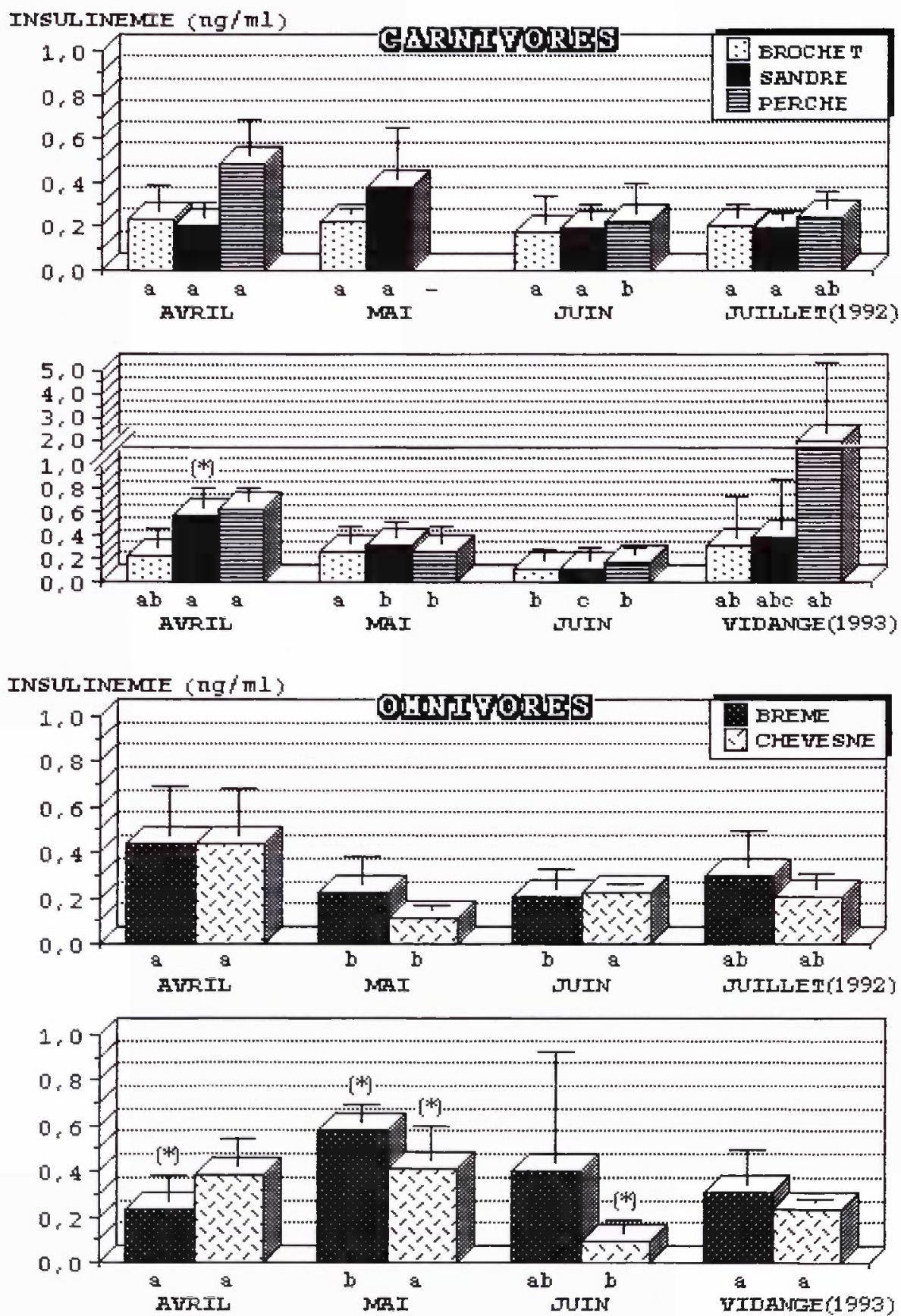

Fig. 8. - Evolution de l'insulinémie au cours de deux périodes. (Annotations, voir Fig. 1).

Fig. 8. - Changes in plasma insulin levels during two periods. (For details, see also Fig. 1). 
teignant environ 20 millions de $\mathrm{m}^{3}$ d'eau au mois de mars, puis 2 millions de $\mathrm{m}^{3}$ en juin et 0,3 millions de $\mathrm{m}^{3}$ juste avant l'ouverture des vannes de fond. Dans ces conditions, on pouvait supposer que la restriction de l'espace vital entraînerait des modifications du comportement chez les poissons, la réduction du plan d'eau au printemps ayant certainement un effet sur la reproduction et aussi sur l'alimentation.

Au cours de cette étude, s'est posé un problème d'échantillonnage. Lors des campagnes de pêches, il a été difficile de recueillir un nombre d'individus suffisant pour chaque espèce et même au cours de la vidange, au niveau de la pêcherie, nous n'avons pas obtenu toutes les espèces souhaitées. Ceci pourrait indiquer que la totalité de la population pisciaire n'a pas été entraînée vers l'aval du barrage (cas des truites, perches, chevesnes...).

II est à noter que la température de l'eau est nettement plus basse en 1993 qu'en 1992; or, ce facteur est déterminant dans le déclenchement de la fraie chez les poissons (Weatherley, 1987; Townsend et Perrow, 1989). La perche et le sandre pondent dans nos régions entre avril et juin, lorsque la température avoisine les $14-15^{\circ} \mathrm{C}$. La fraie des brèmes et des chevesnes nécessite, en général, un minimum de $15^{\circ} \mathrm{C}$, tandis que le brochet peut pondre dès la fin février, dans une eau à $7-10^{\circ} \mathrm{C}$ (Spillman, 1961). On peut penser que, chez les Percidés présentant des rapports gonadosomatiques identiques entre les deux années, la température ne serait pas la seule responsable du moment de la reproduction; les perturbations dues à la diminution de l'espace vital entraîneraient, en 1993, un avancement de l'émission des produits génitaux. Cela se retrouve aussi chez le chevesne chez qui il est difficile d'indiquer, au cours de l'année 1993, le moment de la baisse pondérale des gonades. Par contre, chez la brème et le brochet, la vidange de la retenue ne semble pas avoir d'effet sur la fraie.

Le facteur de condition est une des caractéristiques morphophysiologiques permettant de déceler les changements de poids (Nikolsky, 1963; Velasco et al., 1990) et de déterminer ainsi les phases d'anabolisme ou de catabolisme (Boivin et Power, 1990; Montecchia et al., 1990; Higgs et al., 1992; dos Santos et al., 1993; Jobling et al., 1993; Jorgensen et al., 1993). Habituellement, on observe une baisse de ce facteur au moment de la reproduction puis une hausse jusqu'à la fin de la période d'alimentation (Salinas et al., 1983; dos Santos et al., 1993; Jobling et al., 1993). De même, le rapport hépatosomatique peut rendre compte de l'état nutritionnel des poissons; en effet, l'augmentation pondérale du foie est d'autant plus rapide que la ration alimentaire est importante (Jafri, 1969; Tyler et Dunn, 1976; Bulow et al., 1978; Allen et Wootton, 1982). Entre avril et juillet 1992, le facteur de condition reste relativement stable chez toutes les espèces étudiées, ce qui impliquerait l'existence d'une phase d'alimentation 
intense après la fraie (Karas, 1990; Jobling et al., 1993). Entre avril et juin 1993, les carnassiers présentent des facteurs de condition plus importants; la température étant plus basse, on peut penser que leurs pertes énergétiques sont plus faibles. Ces espèces ne montrent pas, néanmoins, de modifications importantes du rapport hépatosomatique; par conséquent, la concentration des poissons suite à la réduction de l'espace vital ne semble provoquer ni un arrêt de la nutrition ni une augmentation de la prise alimentaire (Jorgensen et al., 1993). Cependant, chez le brochet et le sandre, ce facteur est plus élevé en fin de vidange, laissant supposer une capture des proies facilitée dans 0,3 millions de $\mathrm{m}^{3}$ d'eau. Ainsi, si les carnivores présentent en 1993 un facteur de condition plus élevé, traduisant un état général satisfaisant (Boivin et Power, 1990; Montecchia et al., 1990), le rapport hépatosomatique n'est pas significativement modifié quelles que soient la cote d'eau du bassin ou la température de l'eau. Chez la brème, toutefois, le comportement alimentaire paraît perturbé par la diminution du volume de la retenue; en effet, un grand nombre d'individus avaient le tube digestif totalement vide à la fin de la vidange contrairement aux autres mois. Par ailleurs, de faibles valeurs du facteur de condition étaient enregistrées au même moment. Le changement de la qualité trophique du milieu, à savoir la prolifération, dès le mois d'avril 1993, du phytoplancton pourrait être à l'origine de la baisse du rapport hépatosomatique obser- vée. Hoogenboezem et al. (1992) ont, en effet, signalé que la brème ne s'alimente pas ou bien de façon sporadique lorsque la densité de zooplancton est faible. Par contre, chez le chevesne qui a un régime alimentaire beaucoup plus diversifié, on note une variabilité importante du rapport hépatosomatique, sans qu'il y est de modification marquée du facteur de condition.

De nombreux auteurs (Hille, 1982; Shimma et al., 1982; Miguel et al., 1988; Sandnes et al., 1988; Lemaire et al., 1991) ont signalé, chez diverses espèces de poissons, des fluctuations de la protéinémie, cholestérolémie et triglycéridémie au cours du cycle vital. Cependant, lors d'une étude précédente (Bau et al., 1994), nous avions indiqué que les poissons vivant dans la retenue de Pareloup présentaient une relative stabilité des valeurs de ces paramètres sanguins tout au long du cycle annuel. De plus, la comparaison des deux périodes de référence (1992/1993) ne montre également pas de variations significatives de ces différents composés plasmatiques, ce qui implique que la modification du volume d'eau de la retenue n'influe pas sur ces paramètres, excepté chez le sandre, qui présente en fin de vidange des teneurs élevées en protéines, cholestérol et triglycérides plasmatiques. Cette espèce ne semble donc pas être perturbée, se montrant même opportuniste en augmentant sa prise alimentaire au moment où la concentration des animaux dans la masse d'eau est maximale. 
Les valeurs de l'hématocrite obtenues au cours de cette étude sont comparables à celles trouvées dans la littérature chez diverses espèces de poissons (Perez et al., 1989; Hemre et al., 1990; Pearson et Stevens, 1991). Chez tous les animaux étudiés, l'hématocrite reste relativement constant au cours des deux périodes: il ne paraît varier ni en fonction de la température (Parent et al., 1976; Lane, 1979; Sandnes et al., 1988) ou de la photopériode (Lane, 1979; Sandnes et al., 1988), ni en fonction du régime alimentaire (Zeitoun et al., 1974; Hemre et al., 1990, 1991; Plisetskaya et al., 1991). Si des modifications de ce facteur ont été signalées lors d'un état de stress (Fletcher, 1975; van Vuren et Hattingh, 1978; Witters et al., 1990; Pearson et Stevens, 1991; Wells et Weber, 1991), de telles variations n'apparaissent pas clairement dans cette étude. La restriction progressive de l'espace vital au printemps 1993 et les deux différents types de capture des poissons ne semblent pas modifier significativement l'hématocrite. Cependant, le chevesne présente, à la fin de la vidange, des valeurs élevées de l'hématocrite; on peut penser que l'activité importante de l'animal (Love, 1980; Wells et Weber, 1991), déployée pour remonter vers l'amont, engendrerait une hausse de ce paramètre. Par ailleurs, la grande variabilité des valeurs observée chez le sandre au cours des deux années pourrait s'expliquer par un état asphyxique (Pearson et Stevens, 1991) provoqué par l'accrochage de ces poissons aux filets de pêches. De plus, chez ces animaux, on observe de très fortes teneurs en glucose plasmatique. En effet, les valeurs de la glycémie sont généralement plus élevées que celles données dans la littérature. Le stress, que l'animal subit pendant la capture et le prélèvement sanguin, est certainement responsable de cette hyperglycémie. De nombreux auteurs ont indiqué que la glycémie est reliée au stress de la capture et de la manipulation (Perrier et al., 1971, 1978, 1979; Ince et Thorpe, 1975; Casillas et Smith, 1977; Schwalme et Mackay, 1985, 1990; Hemre et al., 1991). Il a été noté par Perrier (comm. pers.) que les poissons capturés par engins en milieu naturel présentent toujours des valeurs de la glycémie supérieures à celles trouvées chez les animaux "stressés" en laboratoire. Mais il est difficile de donner les raisons de l'apparition de ces très fortes valeurs relevées chez le sandre, un tel taux de glucose sanguin n'ayant jamais été signalé chez un Téléostéen. Aussi, des études complémentaires sont-elles nécessaires pour confirmer ou infirmer l'existence de telles valeurs de glycémie chez les poissons. Cependant, on pourrait relier l'hyperglycémie observée en juillet 1992 aux températures élevées et à la photopériode comme l'ont signalé Haider (1970), Hille (1982), Sweeting et al. (1985) ou Perez et al. (1988). En 1993, les modifications saisonnières de ce facteur sont moins marquées, mais les plus faibles valeurs sont relevées lors de la vidange du plan d'eau. Si les om- 
nivores paraissent, d'une manière générale, moins sensibles d'une part à la restriction de l'espace vital et d'autre part aux modes de capture utilisés, on peut noter que les carnassiers, récupérés à la pêcherie, ont des taux de glucose plasmatique plus bas et proches de ceux trouvés par Perez et al. (1989) ou Gutierrez et al. (1991) chez le bar et Sundby et al. (1991) chez divers Salmonidés, ce qui confirmerait l'augmentation de la glycémie observée lors de la prise aux filets. II semble que l'hyperglycémie chez ces poissons n'ait pas d'effet sur l'insulinosécrétion. De manière générale, les valeurs de l'insulinémie obtenues au cours de cette étude sont très faibles chez toutes les espèces. Par ailleurs, les techniques radio-immunologiques établies pour les mammifères ont été utilisées chez les poissons afin de déterminer le sens de variation de cette hormone. Thorpe et Ince (1976), Emdin et Steiner (1980) ou Furuichi et al. (1980) ont montré que les taux d'insuline évalués par les systèmes homologues sont, chez la même espèce, 4 à 5 fois supérieurs à ceux obtenus par les systèmes hétérologues. Or, actuellement, il nous est difficile d'envisager l'utilisation de techniques de dosages "homologues" pour chaque espèce considérée. Cependant, l'obtention de teneurs en insuline plasmatique plus élevées chez les carnassiers récupérés à la pêcherie laisse à penser que chez les poissons capturés aux filets, les catécholamines libérées à la suite du stress (Delahunty et al., 1978; Carrillo et al.,
1982; Gutierrez et al., 1984; Tilzey et al., $1985 \mathrm{a}, \mathrm{b})$ inhiberaient la sécrétion d'insuline (Wagner et McKeown, 1982; Gapp, 1987).

De ces premiers résultats, il ressort que la variabilité du volume de la retenue ne semble pas avoir d'incidence pathologique majeure sur le métabolisme des poissons. II est fort probable que l'obtention, au niveau de la pêcherie, d'animaux dans un état physiologique correct soit due au fait que la température de l'eau n'a jamais dépassé $15^{\circ} \mathrm{C}$. En effet, le transfert de ces poissons dans d'autres plans d'eau n'a montré que de très faibles mortalités. Cependant, le comportement alimentaire des poissons est différent selon l'espèce considérée. La brème paraît plus sensible aux variations du milieu : à la fin de la vidange, la perturbation de la chaîne trophique (Ferroni et al., 1994) semble avoir, en effet, inhibé la préhension de nourriture. Par contre, chez le chevesne et les carnivores, la concentration du peuplement pisciaire n'a pas perturbé leur prise alimentaire. Mais le fait le plus surprenant sont les très fortes valeurs de la glycémie obtenues chez le sandre; cette hyperglycémie consécutive au stress occasionné lors de la pêche aux filets se retrouve, néanmoins, chez les autres espèces étudiées.

Aussi, pour mieux apprécier l'impact de la vidange sur la physiologie de ces poissons, des analyses biochimiques complémentaires seront-elles réalisées. 


\section{RÉFÉRENCES}

Allen J.R.M. \& Wootton R.J., 1982. Effect of food on the growth of carcass, liver and ovary in the female (Gasterosteus aculeatus L.). J. Fish Biol., 21, 537547.

Bau F., Parent J.P. \& Vellas F., 1994. Evolution saisonnière de paramètres sanguins chez divers Téléostéens capturés dans une retenue. Ichtyophysiologica Acta, 17, 63-89.

Boivin T.G. \& Power G., 1990. Winter condition and proximate composition of anadromous Arctic charr (Salvelinus alpinus) in eastern Ungava bay, Quebec. Can. J. Zool., 68, 2284-2289.

Bulow F.J., Coburn C.B. \& Cobb J.R., 1978. Comparisons of two bluegill populations by means of the RNA-DNA ratio and liver somatic index. Trans. Amer. Fish. Soc., 107, 799-803.

Carrillo M., Zanuy S. \& Herrera E., 1982. Growth and diurnal variations in metabolic parameters in the starved bass, Dicentrarchus labrax, after experimental feeding. Comp. Biochem. Physiol., 72A, 11-16.

Casillas E. \& Smith L.S., 1977. Effect of stress on blood coagulation and haematology in rainbow trout (Salmo gairdneri). J. Fish Biol., 10, 481-491.

Delahunty G., Olcese J., Prack M., Vodicnik M.J., Schreck C.B. \& De Vlaming V.L., 1978. Diurnal variations in the physiology of the goldfish, Carassius auratus L. J. Interdiscipl. Cycle Res., 9, 73-88.

Dos Santos J., Burkow I.C. \& Jobling M., 1993. Patterns of growth and lipid deposition in cod (Gadus morhua L.) fed natural prey and fish-based feeds. Aquaculture, 110, 173-189.

Emdin S.O. \& Steiner D.F., 1980. A specific antiserum against insulin from the Atlantic hagfish, Myxine glutinosa: characterization of the antiserum, its use in a homologous radioimmunoas- say and immunofluorescent microscopy. Gen. Comp. Endocrinol., 42, 251258.

Ferroni J.M., Francisco P., Parent J.P. \& Tourenq J.N., 1994. Relations entre la composition biochimique du plancton et la composition corporelle de deux Téléostéens en milieu lacustre (à paraître).

Fletcher G.L., 1975. The effects of capture, "stress" and storage of whole blood on the red blood cells, plasma proteins, glucose and electrolytes of the winter flounder (Pseudopleuronectes americanus). Can. J. Zool., 53, 197-206.

Furuichi M., Nakamura Y. \& Yone Y., 1980. A radioimmunoassay method for determination of fish plasma insulin. Bull. Japan. Soc. Sci. Fish., 46(9), 11771181.

Gapp D.A., 1987. Endocrine and related factors in the control of metabolism in nonmammalian Vertebrates. In: "Fundamentals of Comparative Vertebrate Endocrinology". Chester-Jones I., Ingleton P.M. et Phillips J.G. Éds, Plenum press, New-York/london, 511-660.

Gutierrez J., Carrillo M., Zanuy S. \& Planas J., 1984. Daily rhythms of insulin and glucose levels in the plasma of sea bass (Dicentrarchus labrax) after experimental feeding. Gen. Comp. Endocrinol., 55, 393-397.

Gutierrez J., Perez J., Navarro I., Zanuy S. \& Carrillo M., 1991. Changes in plasma glucagon and insulin associated with fasting in sea bass (Dicentrarchus labrax). Fish Physiol. Biochem., 9(2), 107-112.

Haider G., 1970. Hämatologische Beobachtungen an Regenbogenforellen ( $\mathrm{Sal}$ mo gairdneri Rich.). -Il. Der Blutzuckerspiegel. Z. Fischerei, 18, 209-216.

Hemre G.I., Lambertsen G. \& Lie O., 1991. The effect of dietary carbohydrate on the stress response in cod 
(Gadus morhua). Aquaculture, 95, 319-328.

Hemre G.I., Lie O., Lambertsen G. \& Sundby A., 1990. Dietary carbohydrate utilization in cod (Gadus morhua). Hormonal response of insulin, glucagon and glucagon-like-peptide to diet and starvation. Comp. Biochem. Physiol., 97A(1), 41-44.

Higgs D.A., Dosanjh B.S., Uin L.M., Himick B.A. \& Eales J.G., 1992. Effects of dietary lipid and carbohydrate levels and chronic 3,5,3'-triiodo-L-thyronine treatment on growth, appetite, food and protein utilization and body composition of immature rainbow trout, Oncorhynchus mykiss, at low temperature. Aquaculture, 105, 175-190.

Hille S., 1982. A literature review of the blood chemistry of rainbow trout, Salmo gairdneri Rich. J. Fish Biol., 20, 535-569.

Hoogenboezem W., Lammens E.H.R.R., Vanvugt Y. \& Osse J.W.M., 1992. A model for switching between particulate-feeding and filter-feeding in the common bream (Abramis brama). Environ. Biol. Fish., 33, 13-22.

Ince B.W. \& Thorpe A., 1975. Hormonal and metabolite effects on plasma free fatty acids in the Northern pike, Esox lucius L. Gen. Comp. Endocrinol., 27, 144-152.

Jafri A.K., 1969. Seasonal changes in the biochemical composition of the freshwater cattish, Wallagonia attu (Bloch.). Hydrobiologia., 72, 497-506.

Jobling M., Jorgensen E.H. \& Siikavuopio S.I., 1993. The influence of previous feeding regime on the compensatory growth response of maturing and immature Arctic charr, Salvelinus alpinus. J. Fish Biol., 43, 409-419.

Jorgensen E.H., Christiansen J.S. \& Jobling M., 1993. Effects of stocking density on food intake, growth performance and oxygen consumption in Arctic charr (Salvelinus alpinus.). Aquaculture, 110, 191-204.
Karas P., 1990. Seasonal changes in growth and standard metabolic rate of juvenile perch (Perca fluviatilis L.). J. Fish Biol., 37, 913-920.

Lane H.C., 1979. Progressive changes in haematology and tissue water of sexually mature trout, Salmo gairdneri Richardson during the autumn and winter. J. Fish Biol., 15, 425-436.

Lemaire P., Drai P., Mathieu A., Lemaire S., Carriere S., Giudicelli J. \& Lafaurie M., 1991. Changes with different diets in plasma enzymes (GOT, GPT, LDH, ALP) and plasma lipids (cholesterol, triglycerides) of sea bass (Dicentrarchus labrax). Aquaculture, 93, 63-75.

Love R.M., 1980. "The chemical biology of fishes, 2". Academic Press, London/New-York, pp. 943.

Miguel J.L., Agapito M.T. \& Recio J.M., 1988. Sex differences of hematological and biochemical parameters in healthy rainbow trout (Salmo gairdneri, R.). Rev. esp. Fisiol., 44(2), 215-220.

Montecchia C.L., Crupkin M. \& Trucco R.E, 1990. Seasonal variations in biochemical and physicochemical properties of actomyosin and energy content of the liver, gonads and muscle of mature argentine hake, Merluccius hubbsi marini. J. Fish Biol., 37, 837-844.

Morgan C.R. \& Lazarow A., 1963. Immunoassay of insulin: two antibody system. Diabetes, 12, 115-126.

Nikolsky G.V., 1963. "The ecology of fishes". Academic Press, London/NewYork, pp. 352.

Parent J.P., Bouche G. \& Vellas F., 1976. Effet du réchauffement artificiel des eaux sur quelques paramètres physiologiques de deux Téléostéens d'eau douce : le gardon et la perche. Cahiers Lab. Hydrobiol. Montereau, 3, 5-14.

Pearson M.P. \& Stevens E.D., 1991. Size and hematological impact of the splenic erythrocyte reservoir in rainbow trout, Oncorhynchus mykiss. Fish Physiol. Biochem., 9(1), 39-50. 
Perez J., Gutierrez J., Carrillo M., Zanuy S. \& Fernandez J., 1989. Effect of bonito insulin injection on plasma immunoreactive glucagon levels and carbohydrate and lipid metabolism of sea bass (Dicentrarchus labrax). Comp. Biochem. Physiol., 94A(1), 3336.

Perez J., Zanuy S. \& Carrillo M., 1988. Effects of diet and feeding time on daily variations in plasma insulin, hepatic c-AMP and other metabolites in a teleost fish, Dicentrarchus labrax L. Fish Physiol. Biochem., 5(4), 191-197.

Perrier H., Perrier C. \& Gras J., 1971. Etude de l'hyperglycémie adrénalique chez la truite arc-en-ciel d'élevage (Salmo gairdneri Richardson). Action des substances adrénolytiques. C.r. Séanc. Soc. Biol., 165, 2141-2144.

Perrier C., Perrier H. \& Terrier M., 1979. Les taux plasmatiques d'AMP cyclique et de glucose chez la truite (Salmo gairdneri Richardson) soumise à diverses formes d'agression. Ichtyophysiologica Acta, 3, 97-103.

Perrier C., Terrier M. \& Perrier H., 1978. A time-course study of the effects of angling stress on cyclic AMP, lactate and glucose plasma levels in the rainbow trout (Salmo gairdneri Richardson) during a 64 hour recovery period. Comp. Biochem. Physiol., 60A, 217219.

Plisetskaya E.M., Buchelli-Narvaez L.I., Hardy R.W. \& Dickhoff W.W., 1991. Effects of injected and dietary arginine on plasma insulin levels and growth of Pacific salmon and rainbow trout. Comp. Biochem. Physiol., 98A(1), 165-170.

Salinas I.M. \& McLaren I.A., 1983. Seasonal variation in weight-specific growth rates, feeding rates and growth efficiencies in Microgadus tomcod. Can. J. Fish. Aquat. Sci., 40, 21972200.

Sandnes K., Lie O. \& Waagbo R., 1988. Normal ranges of some blood chemisry parameters in adult farmed Atlantic salmon, Salmo salar. J. Fish Biol., 32 , 129-136.

Schwalme K. \& Mackay W.C., 1985. The influence of angling-induced exercise on the carbohydrate metabolism of northern pike (Esox lucius L.). J. Comp. Physiol., 156B, 67-75.

Schwalme K. \& Mackay W.C., 1990. Mechanisms that elevate the glucose concentration of muscle and liver in yellow perch (Perca flavescens Mitchill) after exercise-handling stress. Can. J. Zool., 69, 456-461.

Shimma Y., Shimma H. \& Ikeda K., 1982. Plasma constituents of 1-year-old rainbow trout raised with fish meal and SCP combined feeds. Bull. Japan. Soc. Sci. Fish., 48(6), 805-810.

Spillman C.J., 1961. Poissons d'eau douce. In: "Faune de France, 65 ». Lechevalier P. Éds., Paris, pp. 304.

Sundby A., Eliassen K., Refstie T. \& Plisetskaya E.M., 1991. Plasma levels of insulin, glucagon and glucagon-like peptide in salmonids of different weights. Fish Physiol. Biochem., 9(3), 223-230.

Sweeting R.M., Wagner G.F. \& McKeown B.A., 1985. Changes in plasma glucose, amino acid nitrogen and growth hormone during smoltification and seawater adaptation in coho Salmon, Oncorhynchus kisutch. Aquaculture, 45, 185-197.

Thorpe A. \& Ince B.W., 1976. Plasma insulin levels in teleosts as determined by a charcoal-separation radioimmunoassay technique. Gen. Comp. Endocrinol., 30, 332-339.

Tilzey J.F., Waights V. \& Holmes R., 1985a. The development of a homologous teleost insulin radioimmunoassay and its use in the study of adrenaline on insulin secretion from isolated pancreatic islet tissue of the rainbow trout, Salmo gairdneri (R.). Comp. Biochem. Physiol., 81A(4), 821-825. 
Tilzey J.F., Waights V. \& Holmes R., 1985b. Adrenergic control of insulin release from isolated islet tissue in the rainbow trout, Salmo gairdneri (R.). Gen. Comp. Endocrinol., 59, 460-467.

Townsend C.R. \& Perrow M.R., 1989. Eutrophication may produce population cycles in roach, Rutilus rutilus (L.), by two contrasting mechanisms. J. Fish Biol., 34, 161-164.

Tyler A.V. \& Dunn R.S., 1976. Ration growth and measures of somatic and organ condition in relation to meal frequency in winter flounder (Pseudopleuronectes americanus) with hypothese regarding population homeostasis. J. Fish. Res. Board. Can., 33, 63-75.

Van Vuren J.H.J. \& Hattingh J., 1978. A seasonal study of the haematology of wild freshwater fish. J. Fish Biol., 13, 305-313.

Velasco J.C., Rincon P.A. \& Lobon-Cervia J., 1990. Age, growth and reproduction of the cyprinid Rutilus lemmingii (Steindachner, 1866) in the River Huebra, Central Spain. J. Fish Biol., 36, 469-480.

Wagner G.F. \& McKeown B.A., 1982. Changes in plasma insulin and carbo- hydrate metabolism of zinc-stressed rainbow trout, Salmo gairdneri. Can. J. Zool., 60, 2079-2084.

Weatherley N.S., 1987. The diet and growth of 0-group dace, Leuciscus leuciscus (L.) and roach, Rutilus rutilus (L.), in a lowland river. J. Fish Biol., 30, 237-247.

Wells R.M.G. \& Weber R.E., 1991. Is there an optimal haematocrit for rainbow trout, Oncorhynchus mykiss (Walbaum)? An interpretation of recent data based on blood viscosity measurements. J. Fish Biol., 38, 5365.

Witters H.E., Van Puymbroeck S., Van den Sande I. \& Vanderborght O.L.J., 1990. Haematological disturbances and osmotic shifts in rainbow trout, Oncorhynchus mykiss (Walbaum) under acid and aluminium exposure. J. Comp. Physiol., 160B, 563-571.

Zeitoun I.H., Ullrey D.E. \& Tack P.I., 1974. Effects of water salinity and dietary protein levels on total serum protein and hematocrit of rainbow trout (Salmo gairdneri) fingerlings. J. Fish. Res. Board Can., 31, 1133-1134. 